\title{
Identification of the key genes and long non-coding RNAs in ankylosing spondylitis using RNA sequencing
}

\author{
ZHENGKUAN XU, HAO LI, QIXIN CHEN and GANG CHEN \\ Department of Orthopedics, 2nd Affiliated Hospital, School of Medicine, \\ Zhejiang University, Hangzhou, Zhejiang 310009, P.R. China
}

Received April 12, 2018; Accepted December 7, 2018

DOI: $10.3892 /$ ijmm.2018.4038

\begin{abstract}
Ankylosing spondylitis (AS) is an insidious and debilitating form of arthritis that involves the axial skeleton, and its etiology and pathogenesis remain unclear. In the present study, three patients with AS and three normal controls from our hospital were enrolled. RNA sequencing and bioinformatics analysis were performed in order to identify the differentially expressed (DE) mRNAs (DEmRNAs) and DE long non-coding RNAs (DElncRNAs) between the patients with AS and normal controls. Construction of an AS-specific protein-protein interaction network, a weighted DElncRNA-DEmRNA co-expression network and functional annotation of the DEmRNAs co-expressed with DElncRNAs was performed.Nearby cis-targeted DEmRNAs or DElncRNAs were identified by searching for DEmRNAs that were transcribed within 100-kb up- or downstream of DElncRNAs. Based on the Gene Expression Omnibus datasets GSE25101 and GSE73754, the expression of selected DEmRNAs and DElncRNAs were verified using published RNA sequencing data from blood samples, and receiver operating characteristic analysis of selected DEmRNAs was performed. Compared with the normal controls, 1,072 DEmRNAs and 372 DElncRNAs in the patients with AS were identified. Caspase recruitment domain family member 11 and DNA methyltransferase 1 have great diagnostic value for AS. MSTRG.8559 and LINC00987 were also identified as two hub DElncRNAs. The T-cell receptor signaling pathway was a significantly enriched pathway of the DEmRNAs co-expressed with DElncRNAs in patients with AS. In conclusion, the present study identified
\end{abstract}

Correspondence to: Dr Gang Chen, Department of Orthopedics, 2nd Affiliated Hospital, School of Medicine, Zhejiang University, 88 Jie Fang Road, Hangzhou, Zhejiang 310009, P.R. China

E-mail: chengang1976@zju.edu.cn

Abbreviations: AS, ankylosing spondylitis; DEmRNAs, differentially expressed mRNAs; DElncRNAs, differentially expressed long non-coding RNAs; GO, Gene Ontology; KEGG, Kyoto Encyclopedia of Genes and Genomes; PPI, protein-protein interaction

Key words: ankylosing spondylitis, RNA sequencing, lncRNAs the key DEmRNAs and DElncRNAs in AS, which provides novel information for understanding the pathogenesis of AS and developing potential biomarkers for AS.

\section{Introduction}

Ankylosing spondylitis (AS) is the prototypic and debilitating type of spondyloarthritis, which is an autoimmune disorder (1). AS often affects the axial joints, including the sacroiliac joint and spine, and induces new bone formation and ultimately ankylosis $(2,3)$.

AS is associated with the interplay of genetic risks and environmental triggers, and its etiology and pathogenesis remain largely unknown. A number of studies have reported a strong association between the major histocompatibility complex class I allele human leukocyte antigen B27 (HLA-B27) and AS (4-6). However, the mechanism by which HLA-B27 causes a predisposition to AS remains unclear. T cells and a number of immune pathways, including the interleukin (IL)-17/IL-23 pathway and control of nuclear factor $-\kappa \mathrm{B}(\mathrm{NF}-\kappa \mathrm{B})$ activation, have been reported to be closely associated with AS $(5,6)$. Currently, it is difficult to diagnose AS during the early stages due to the lack of accurate diagnostic biomarkers.

Long non-coding RNA (lncRNA) is a type of non-proteincoding transcript with a length of $>200$ nucleotides; they are emerging as key regulators in various biological processes (7). Recent studies have indicated that lncRNAs, including lncRNA-AK001085, lnc-zinc finger protein 354A (ZNF354A)-1, lnc-Lin-54 DREAM MuvB core complex component (LIN54)-1, lnc-Facioscapulohumeral muscular dystrophy region gene 2 family member C (FRG2C)-3 and lnc-ubiquitin specific peptidase 50 (USP50)-2, serve roles in AS $(8,9)$. Some researchers have also revealed that mRNAs, such as programmed cell death 1 (PDCD1) (1), caspase recruitment domain-containing protein 11 (CARD11) (10), phospholipase $\mathrm{C} \gamma 1$ (PLCG1) $(11,12)$ and DNA methyltransferase 1 (DNMT1) (13), may be involved in the pathogenesis of AS.

In the present study, the key differentially expressed (DE) mRNAs (DEmRNAs) and DElncRNAs in AS were identified using RNA sequencing and bioinformatics analysis. DElncRNA-DEmRNA co-expression network construction, identification of nearby target DEmRNAs of DElncRNAs and functional annotation of DEmRNAs were performed in order 
to understand the biological functions of the key DEmRNAs and DElncRNAs in AS.

\section{Materials and methods}

Patients and samples. Three patients with AS and three normal controls were enrolled in the present study from 2 nd Affiliated Hospital, School of Medicine, Zhejiang University (Zhejiang, China). The patients with AS were aged 37, 36 and 40 years old, and all were HLA-B27 $7^{+}$and male. These patients were diagnosed with AS based on kyphotic deformity, bilateral damage of the sacroiliac joint observed in the computed tomography results, and spinal fusion and sacroiliac arthrodesis observed in the X-ray results. All of the patients had not received treatment with non-steroidal anti-inflammatory drugs or biologics and they had not exhibited complications. The normal controls were aged 35, 36 and 37 years old and were male. None of the six participants had any other type of autoimmune disease. Blood samples were obtained from all six participants. All of the participants submitted written informed consent and the present study was approved by the Ethics Committee of 2nd Affiliated Hospital, School of Medicine, Zhejiang University.

Library preparation and high-throughput sequencing. Total RNA was isolated from the blood samples with TRIzol reagent (Invitrogen; Thermo Fisher Scientific, Inc., Waltham, MA, USA) according to the manufacturer's instructions. A NanoDrop ND-2000 spectrophotometer (Thermo Fisher Scientific, Inc.) was used to check the concentration and purity of the RNA. The integrity of the RNA was confirmed using $2 \%$ agarose gel and the RNA integrity number (RIN) was obtained using an Agilent 2100 Bioanalyzer instrument (Agilent Technologies, Inc., Santa Clara, CA, USA). The thresholds of total RNA for cDNA library construction were as follows: i) Amount of RNA, $>5 \mu \mathrm{g}$; ii) concentration of RNA; $\geq 200 \mathrm{ng} / \mathrm{ml}$; iii) $1.8<$ optical density (OD) $260 / 280<2.2$; iv) $2.0<\mathrm{OD}_{260 / 280}$; and v) $\mathrm{RIN}$ value, $>8.0$.

Firstly, the ribosomal RNA was removed from the total RNA using a Ribo-Zero Magnetic kit (Epicentre; Illumina, Inc., San Diego, CA, USA). In addition, 'Soap' software (version 1.03; soap.genomics.org.cn/SOAPdenovo-Trans. html\#intro2) was used to compare the reads of the rRNA, and then to write a perl script to remove it from the FastQ file. Subsequently, the cDNA library for RNA sequencing was contrasted using the TruSeq RNA Sample Prep kit (Illumina, Inc.). Briefly, the retrieved RNA was fragmented by adding First Strand Master Mix and then first-strand cDNA was generated using the First Strand Master mix and Super Script II reverse transcription (Invitrogen; Thermo Fisher Scientific, Inc.) with the following reaction conditions: $25^{\circ} \mathrm{C}$ for $10 \mathrm{~min}$, $42^{\circ} \mathrm{C}$ for $50 \mathrm{~min}$ and $70^{\circ} \mathrm{C}$ for $15 \mathrm{~min}$. Following purification of the product with Agencourt RNA Clean XP beads (Beckman Coulter, Inc., Brea, CA, USA), Second Strand Master mix and dATP, dGTP, dCTP and dUTP mix (Beckman Coulter, Inc.) were added to synthesize the second-strand cDNA $\left(16^{\circ} \mathrm{C}\right.$ for $1 \mathrm{~h})$. Subsequently, the purified cDNA was combined with End Repair Mix (Thermo Fisher Scientific, Inc.) and incubated at $30^{\circ} \mathrm{C}$ for $30 \mathrm{~min}$. Following purification with beads (Qiagen, Inc., Valencia, CA, USA), A-Tailing mix (Qiagen, Inc.) was added into the reaction system, which was incubated at $37^{\circ} \mathrm{C}$ for 30 min. Adenylate 3' Ends DNA, Index Adapter and Ligation mix (Qiagen, Inc.) were combined and incubated at $30^{\circ} \mathrm{C}$ for $10 \mathrm{~min}$. Subsequently, the Uracil-N-Glycosylase enzyme was added into the purified ligation product and incubated at $37^{\circ} \mathrm{C}$ for $10 \mathrm{~min}$. A total of 15 rounds of polymerase chain reaction (PCR) amplification were conducted with PCR Primer Cocktail (Illumina, Inc.) and PCR Master Mix (Illumina, Inc.) to enrich the cDNA fragments. The PCR products were then purified with AMPure XP beads (Qiagen, Inc.). The qualified libraries were amplified on cBot (Illumina, Inc.) to generate a cluster on the flow cell (TruSeq PE Cluster kit v3-cBot-HS; Illumina, Inc.). The amplified flow cell was sequenced on an Illumina HiSeq X Ten platform (Illumina, Inc.).

Quality control of raw sequence. Quality control of the raw reads derived from the RNA sequencing was performed to obtain clean reads of high quality. Briefly, quality control involved trimming low-quality reads, including adaptor sequences, sequences with a quality score $<20$ and sequences with an N-base rate of raw reads $>10 \%$, using SeqPrep (version 1.2; github.com/jstjohn/SeqPrep) and Sickle (version V3.4.0; github.com/najoshi/sickle).

Clean reads mapping. Sequences were aligned to the human reference genome GRCh38.p7 (Ensembl v84; www.ensembl. org/index.html) using hierarchical indexing for the spliced alignment of the transcripts programme HISAT2 (version 2.1.0; ccb.jhu.edu/software/hisat2/index.shtml). Then, StringTie (version v1.3.4; ccb.jhu.edu/software/stringtie/) was used to assemble and quantify the transcripts in each sample. Ultimately, differential gene expression analysis was performed with Ballgown (version 3.7; www.bioconductor. org/packages/release/bioc/html/ballgown.html) in R environment.

Identification of DEmRNAs and DElncRNAs. Using Ballgown, the DEmRNAs and DElncRNAs between the patients with AS and normal controls were identified with $\mathrm{P}<0.05$. The false discovering rate (FDR)-adjusted P-value of the test statistic was used. Hierarchical clustering of the DElncRNAs and DEmRNAs expression profile was performed using hcluster in R language (version 3.3.3; stat.ethz.ch/R-manual/ R-devel/library/stats/html/hclust.html).

$A S$-specific protein-protein interaction (PPI) network construction. PPI networks of the top 30 up- and downregulated DEmRNAs, respectively, were constructed using BioGRID (version 3.5; thebiogrid.org).

$A S$-specific weighted DEmRNA-DElncRNA co-expression network analyses. Weighted Gene Co-expression Network Analysis (WGCNA_1.64-1; horvath.genetics.ucla.edu/html/ Coexpres sionNetwork/Rpackages/WGCNA/) (14) is an R package for weighted correlation network analysis, which is also known as weighted gene co-expression network analysis. Using WGCNA, AS-specific weighted DEmRNA-DElncRNA co-expression network analysis was performed. The pairwise Pearson's correlation coefficients (PCCs) between the DElncRNAs and DEmRNAs in patients with AS were calcu- 
Table I. Top 10 up- and downregulated DEmRNAs between ankylosing spondylitis and normal controls.

\begin{tabular}{|c|c|c|c|c|c|}
\hline DEmRNAs & $\log 2$. Fold change & P-value & SD_fpkm_C & SD_fpkm_T & Regulation \\
\hline IGHG1 & -2.021979095 & 0.047255959 & 8.80361236 & 0.515381703 & Down \\
\hline IGHG4 & -1.859252007 & 0.025210597 & 3.368882506 & 0.227307135 & Down \\
\hline TRAJ18 & -1.643179443 & 0.035866398 & 10.07677638 & 4.09159482 & Down \\
\hline TRAJ11 & -1.524711275 & 0.014505975 & 8.654159887 & 4.620202967 & Down \\
\hline TRAJ5 & -1.49467071 & 0.000440647 & 6.737567008 & 2.426348758 & Down \\
\hline TRAJ44 & -1.46271809 & 0.001143618 & 4.42364856 & 1.848651282 & Down \\
\hline ETS1 & -1.335519855 & 0.031765089 & 32.5763664 & 9.312805914 & Down \\
\hline LAIR2 & -1.302776176 & 0.044790098 & 0.834353066 & 1.050550336 & Down \\
\hline ESYT1 & -1.259200819 & 0.002673524 & 15.14634661 & 6.658023234 & Down \\
\hline NCR3 & -1.247053856 & 0.033379705 & 5.641796936 & 2.719067002 & Down \\
\hline CLC & 1.194865831 & 0.043003696 & 21.60725157 & 83.0330712 & Up \\
\hline S100A12 & 0.98788469 & 0.00518168 & 40.98384277 & 110.0364144 & Up \\
\hline CMPK2 & 0.904925259 & 0.01474919 & 0.622597776 & 2.021786682 & Up \\
\hline ZFP36 & 0.842014426 & 0.012071156 & 6.481954564 & 7.910723637 & Up \\
\hline MME & 0.746984037 & 0.031312145 & 9.013396028 & 20.24882244 & Up \\
\hline LIN7A & 0.711395439 & 0.049387957 & 3.456739121 & 4.043733587 & Up \\
\hline CYP4F3 & 0.707298362 & 0.025967428 & 4.648357447 & 8.950444777 & Up \\
\hline CXCL8 & 0.651965182 & 0.048148598 & 6.882829746 & 11.04384805 & Up \\
\hline CLEC4D & 0.636486225 & 0.047074356 & 2.579585737 & 3.692774365 & $\mathrm{Up}$ \\
\hline FAM118A & 0.634156346 & 0.010715671 & 0.992441488 & 2.574184644 & Up \\
\hline
\end{tabular}

DEmRNAs, differentially expressed mRNAs; fpkm, fragments per kilobase million; C, control group; T, case group.

Table II. Top 10 up- and downregulated DElncRNAs between the patients with ankylosing spondylitis and the normal controls.

\begin{tabular}{|c|c|c|c|c|c|}
\hline DElncRNAs & log2.Fold change & P-value & SD_fpkm_C & SD_fpkm_T & Regulation \\
\hline MSTRG.9221 & -2.900321700 & 0.018550654 & 4.278074312 & 0.276811741 & Down \\
\hline MSTRG.1368 & -2.619486415 & 0.003067593 & 1.673345961 & 0 & Down \\
\hline AL928768.3 & -2.320417178 & 0.018613919 & 1.432426837 & 0.766958784 & Down \\
\hline WDR86-AS1 & -1.749482818 & 0.045879379 & 5.820719606 & 0.430167793 & Down \\
\hline GS1-393G12.12 & -1.595574045 & 0.025413087 & 2.892968281 & 0.573066828 & Down \\
\hline RP11-75C10.7 & -1.578454562 & 0.028918409 & 1.681378986 & 0.27755832 & Down \\
\hline MSTRG.22984 & -1.515487489 & 0.028151217 & 6.307372767 & 6.856887292 & Down \\
\hline RP1-29C18.8 & -1.491219357 & 0.007182308 & 1.813751882 & 0.780203396 & Down \\
\hline RP11-20I20.4 & -1.489870084 & 0.045895116 & 1.882568915 & 0.702213888 & Down \\
\hline CTD-2531D15.5 & -1.452569255 & 0.044459184 & 1.198212257 & 0 & Down \\
\hline AC010084.1 & 1.812545185 & 0.005654266 & 0 & 0.808113485 & Up \\
\hline PSMD5-AS1 & 1.788651473 & 0.011076928 & 8.927660428 & 20.42027077 & Up \\
\hline RP11-535M15.1 & 1.639437785 & 0.00820557 & 0 & 1.477784502 & Up \\
\hline LLPH-AS1 & 1.498657545 & 0.005471274 & 0.230318651 & 2.298519226 & Up \\
\hline RP4-811H24.9 & 1.452389476 & 0.004968106 & 0.053490925 & 0.499762058 & $\mathrm{Up}$ \\
\hline MSTRG.30231 & 1.342076952 & 0.040559246 & 2.874551067 & 6.364192991 & Up \\
\hline TMEM92-AS1 & 1.339293051 & 0.014889801 & 0 & 1.352052251 & Up \\
\hline WI2-87327B8.2 & 1.289651543 & 0.017494757 & 1.241932483 & 1.714464098 & Up \\
\hline AC099668.5 & 1.25464443 & 0.023121561 & 0 & 1.059053016 & Up \\
\hline LINC01151 & 1.231390756 & 0.00790838 & 0.384761558 & 0.385097694 & Up \\
\hline
\end{tabular}

DElncRNA, differentially expressed long non-coding RNA; fpkm, fragments per kilobase million; C, control group; T, case group. 


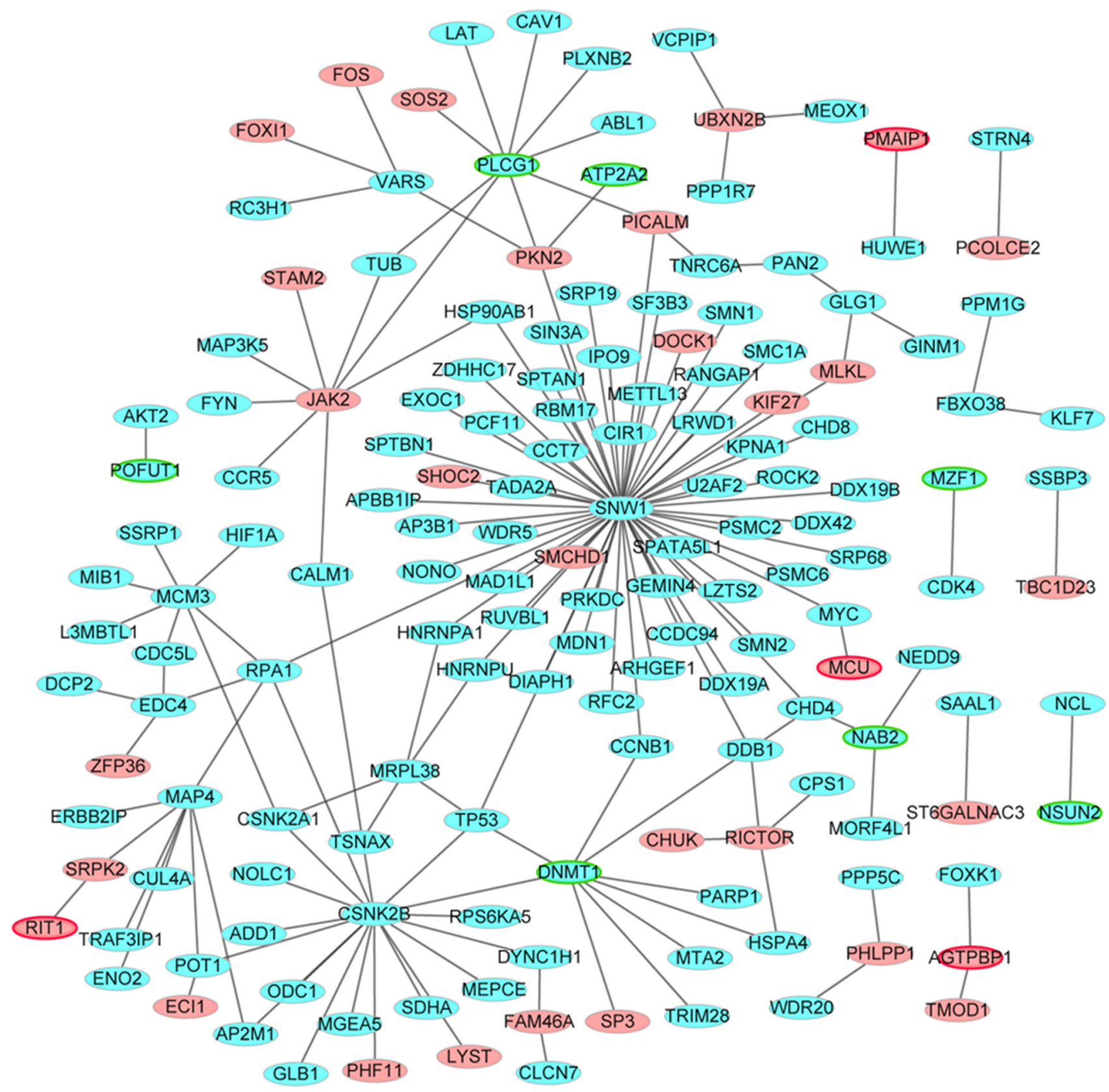

Figure 1. AS-specific PPI network. Blue and red shapes represent proteins encoded by down- and upregulated DEmRNAs, respectively, when comparing patients with AS and normal controls. Among these, blue shapes with green outlines represent proteins encoded by the top 10 downregulated DEmRNAs; red shapes with red outlines represent proteins encoded by the top 10 upregulated DEmRNAs. AS, ankylosing spondylitis; DEmRNA, differentially expressed mRNA.

lated. DElncRNA-DEmRNA pairs with IPCC value $\geq 0.90 \mid$ and $\mathrm{P}<0.001$ were used to construct an AS-specific weighted DEmRNA-DElncRNA co-expression network, which was deciphered using the online-based software GeneCodis3 (genecodis.cnb.csic.es/analysis).

Functional analyses of DEmRNAs co-expressed with DElncRNAs in AS. The DEmRNAs of these DElncRNA-DEmRNA pairs with IPCC value $\geq 0.901$ and $\mathrm{P}<0.001$ were used to conduct Gene Ontology (GO; www. geneontology.org/) and Kyoto Encyclopedia of Genes and Genomes (KEGG; www.genome.jp/kegg/) molecular pathway enrichment analysis using GeneCoDis3.
DEmRNAs close to DElncRNAs with cis-regulatory effects. Previous studies have reported that lncRNAs can regulate genes that are transcribed near to them, consistent with activity in cis-regulatory elements $(15,16)$. Therefore, in the present study DEmRNAs that were close cis targets of DElncRNAs were identified by searching for DEmRNAs that were transcribed within a 100-kb window up- or downstream of DElncRNAs between the patients with AS and the normal controls (17).

Validation of the expression of DEmRNAs and DElncRNAs using the GSE73754 and GSE25101 datasets. The mRNA expression profile of 52 patients with AS and 20 normal controls (Canadian cohort) in the GSE73754 dataset (GPL10558 Illumina 
Table III. The top 100 co-expressed differentially expressed mRNA-differentially expressed long non-coding RNA interaction network.

\begin{tabular}{lll}
\hline mRNAsig & lncRNAsig & corsig
\end{tabular}

OR5B2
ADAMTS14

$\begin{array}{lll}\text { RP11-524O1.4 } 0.999999784 & 7.02 \times 10^{-14}\end{array}$

ADAMTS14 RP5-906A24.2 $0.9999988551 .97 \times 10^{-12}$

DMRTC2

PRY

CAMSAP3

GPR62

ACTN3

COL21A1

OR5B2

SFTA2

ADAMTS14

DMRTC2

FOXI1

FOXI1

ERICH6

DMRTC2

FOXI1

OR2C3

FOXI1

PAK6

DMRTC2

CAMSAP3

GPR62

SFTA2

ADAMTS14

ERICH6

COL21A1

OR2C3

FOXI1

SFTA2

PRY

PRY

OR2C3

PRY

FCRL4

ADAMTS14

PRY

ACTN3

FZD9

RP11-505K9.4

ADAMTS14

FZD9

FZD9

FOXI1

FZD9

PDZD4

ACTN3

KCNMB2-AS1

$0.99999619 \quad 2.18 \times 10^{-11}$

RP11-756H6.1

$0.9999959 \quad 2.52 \times 10^{-11}$

RP11-355N15.3

$0.9999955382 .99 \times 10^{-11}$

LINC01337

LINC01337

AC004471.9

LINC01337

RP11-91I20.2

AC004471.9

$0.9999939525 .49 \times 10^{-11}$

$0.9999939315 .52 \times 10^{-11}$

$0.9999850333 .36 \times 10^{-10}$

$0.9999847423 .49 \times 10^{-10}$

TSPEAR-AS1

$0.9999843 \quad 3.70 \times 10^{-10}$

$0.999975676 \quad 8.88 \times 10^{-10}$

TSPEAR-AS1

$0.9999481894 .03 \times 10^{-9}$

RP5-906A24.2

$0.9999365486 .04 \times 10^{-9}$

AC022431.3

$0.9998888141 .85 \times 10^{-8}$

RP1-72A23.4

$0.999855298 \quad 3.14 \times 10^{-8}$

KCNMB2-AS1

$0.9998539763 .20 \times 10^{-8}$

KCNMB2-AS 1

$0.99982545 \quad 4.57 \times 10^{-8}$

RP11-355N15.3

$0.999815958 \quad 5.08 \times 10^{-8}$

RP11-320N21.1

$0.999806865 \quad 5.59 \times 10^{-8}$

PSMD5-AS 1

$0.999759427 \quad 8.68 \times 10^{-8}$

RP5-906A24.2

$0.9997430049 .91 \times 10^{-8}$

RP11-254I22.2

$0.999736476 \quad 1.04 \times 10^{-7}$

$0.999726506 \quad 1.12 \times 10^{-7}$

RP11-254I22.2

RP11-320N21.1

$0.99972637 \quad 1.12 \times 10^{-7}$

$0.99970509 \quad 1.30 \times 10^{-7}$

RP11-756H6.1

$0.9997045631 .31 \times 10^{-7}$

RP11-254I22.2

$0.999685951 \quad 1.48 \times 10^{-7}$

$0.999676565 \quad 1.57 \times 10^{-7}$

RP11-254122.

RP11-524O1.4

$0.999662773 \quad 1.71 \times 10^{-7}$

TSPEAR-AS1

$0.999646107 \quad 1.88 \times 10^{-7}$

AC022431.3

$0.999573412 \quad 2.73 \times 10^{-7}$

RP11-203E8.1

$0.9995495643 .04 \times 10^{-7}$

RP11-104N10.1

RP11-91I20.2

$0.999534887 \quad 3.24 \times 10^{-7}$

$0.9995221113 .43 \times 10^{-7}$

RP4-736L20.3

$0.999509557 \quad 3.61 \times 10^{-7}$

CTD-2534I21.8

$0.999489932 \quad 3.90 \times 10^{-7}$

AC022431.3

$0.9994410164 .69 \times 10^{-7}$

AC008781.7

$0.999421699 \quad 5.02 \times 10^{-7}$

RP11-320N21.1

AC008781.7

$0.999325423 \quad 6.82 \times 10^{-7}$

$0.999315145 \quad 7.03 \times 10^{-7}$

RP11-756H6.1

$0.999273631 \quad 7.91 \times 10^{-7}$

RP11-320N21.1

$0.999264308 \quad 8.12 \times 10^{-7}$

RP4-736L20.3

$0.999211985 \quad 9.31 \times 10^{-7}$

RP11-104N10.1

$0.99917912 \quad 1.01 \times 10^{-6}$

RP11-756H6.1

$0.999170485 \quad 1.03 \times 10^{-6}$

RP11-203E8.1

$0.99915935 \quad 1.06 \times 10^{-6}$

RP11-159N11.4

$0.9991410061 .11 \times 10^{-6}$

RP11-219A15.1

AC022431.3

$0.9991325021 .13 \times 10^{-6}$

CAMSAP3

Table III. Continued.

\begin{tabular}{llll}
\hline mRNAsig & \multicolumn{1}{c}{ lncRNAsig } & \multicolumn{1}{c}{ corsig } & pvalsig \\
\hline GPR62 & RP1-72A23.4 & 0.999004022 & $1.49 \times 10^{-6}$ \\
OR5B2 & RP11-355N15.3 & 0.998989141 & $1.53 \times 10^{-6}$ \\
ERICH6 & LINC01337 & 0.998988895 & $1.53 \times 10^{-6}$ \\
CXXC5 & LINC01588 & 0.998981114 & $1.56 \times 10^{-6}$ \\
FZD9 & RP1-72A23.4 & 0.998951532 & $1.65 \times 10^{-6}$ \\
COL21A1 & RP1-72A23.4 & 0.99891675 & $1.76 \times 10^{-6}$ \\
NDUFC2- & RP11-45A17.2 & -0.998877807 & $1.89 \times 10^{-6}$
\end{tabular}

\section{KCTD14}

MAP4

PRY

SRGN

MSTRG.8559

$0.998862021 \quad 1.94 \times 10^{-6}$

$0.998818651 \quad 2.09 \times 10^{-6}$

RP11-505K9.4 TSPEAR-AS1

OR2C3

OR2C3

FOXI1

PRY

ZNF804A

FAM111A

OR2C3

DMRTC2

OR2C3

HHAT

IL18BP

RP11-203E8.1

0.998661182

$2.69 \times 10^{-6}$

$0.9986354842 .79 \times 10^{-6}$

$0.9986139362 .88 \times 10^{-6}$

RP11-104N10.1 $0.998588286 \quad 2.99 \times 10^{-6}$

AC004471.9

0.998585363

$3.00 \times 10^{-6}$

RP11-91I20.2

0.998566098

$3.08 \times 10^{-6}$

$0.99856452 \quad 3.09 \times 10^{-6}$

TCEAL3-AS1

0.998552192

0.998544416

$3.14 \times 10^{-6}$

RP4-736L20.3

AC022431.3

RP5-862P8.2

AC008781.7

AC012074.2

SMAD6

CFAP20

MSTRG.8559

0.998494339

$3.18 \times 10^{-6}$

0.99839571

$3.40 \times 10^{-6}$

0.998323875

$3.86 \times 10^{-6}$

0.998273402

$4.21 \times 10^{-6}$

0.998269298

0.998257122

$4.47 \times 10^{-6}$

RP11-1H8.5

RP11-505K9.4

0.998252424

$4.49 \times 10^{-6}$

RP5-1092A3.5

MSTRG.8559

0.998220553

$4.55 \times 10^{-6}$

DMRTC2

PCOLCE2

SFTA2

FZD9

ARL11

MYOF

NT5C2

MME

RP11-505K9.4

IL18BP

HSPA1L

SAMHD1

SFTA2

CSNK2B

NOLC1

TNXB

RHOB

DDB1

ORMDL3

OR5B2

ADAMTS14

TRIM73

0.998212166

$4.58 \times 10^{-6}$

RP11-320N21.1

RP11-105C19.2

$-0.998176846$

$4.75 \times 10^{-6}$

RP5-906A24.2

0.998131862

$4.79 \times 10^{-6}$

RP11-254I22.2

0.998116114

$4.98 \times 10^{-6}$

DNAJC3-AS1

0.998052713

$5.23 \times 10^{-6}$

LINC01151

RP11-139H15.6

$-0.998035735$

$5.32 \times 10^{-6}$

0.998022562

$5.68 \times 10^{-6}$

RP11-295I5.4

0.998001494

$5.78 \times 10^{-6}$

RP5-906A24.2

0.99795661

$5.86 \times 10^{-6}$

MYCBP2-AS2

0.997935664

0.997924492

$5.99 \times 10^{-6}$

LINC01588

MYCBP2-AS2

0.997902735

0.997861652

$6.26 \times 10^{-6}$

KCNMB2-AS1

0.997833473

0.997817608

$6.39 \times 10^{-6}$

$6.46 \times 10^{-6}$

$6.59 \times 10^{-6}$

RP11-214K3.23

MSTRG.8559

RP11-441F2.2

0.997791211

$6.85 \times 10^{-6}$

$7.04 \times 10^{-6}$

LINC00671

0.997708778

$7.14 \times 10^{-6}$

RP11-429J17.2

0.997652855

$7.31 \times 10^{-6}$

$7.87 \times 10^{-6}$ 


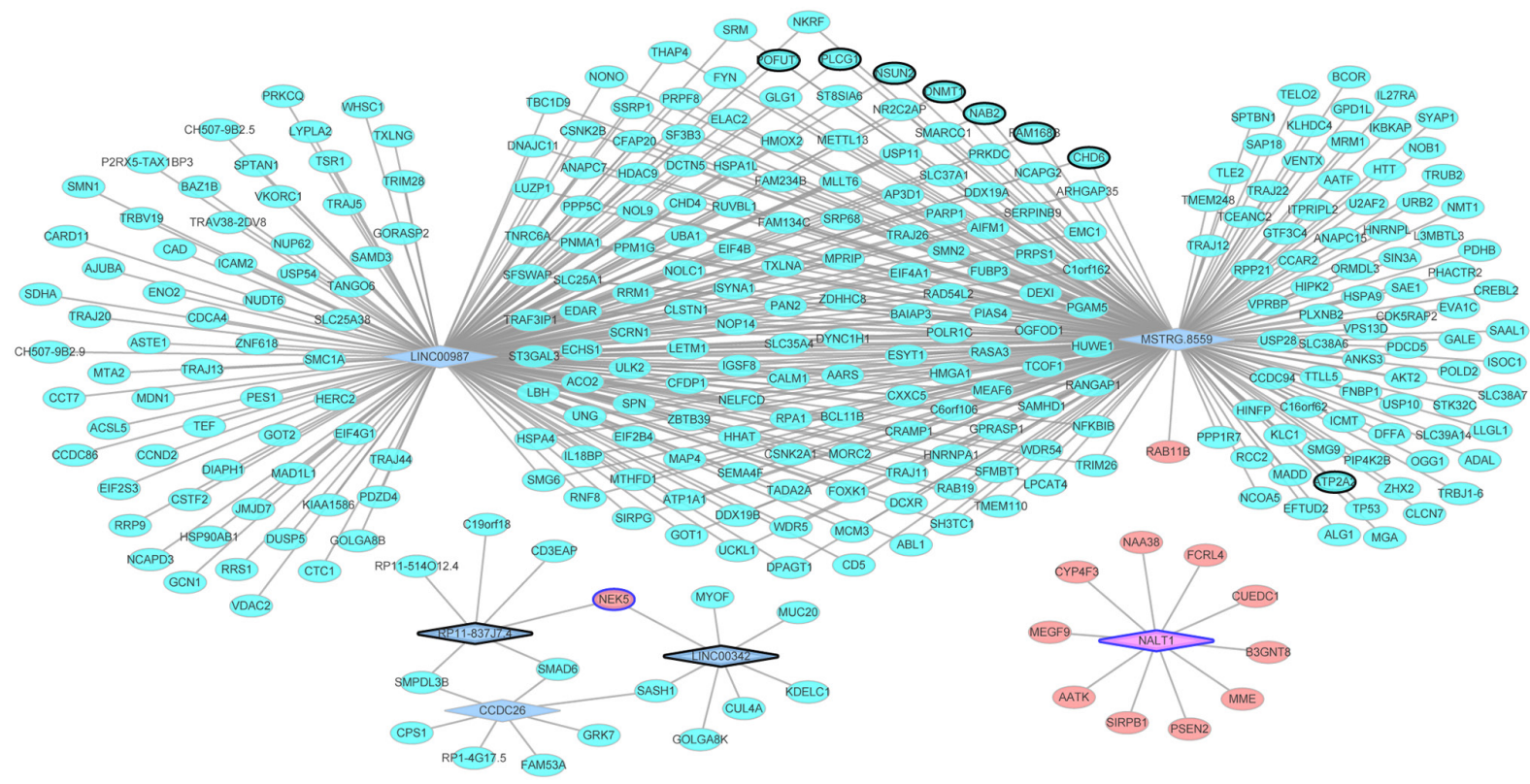

Figure 2. Selected DElncRNA-DEmRNA co-expression network. Ovals and rhombuses represent DEmRNAs and DElncRNAs, respectively, comparing AS patients and normal controls. Shapes with bold black outlines indicate the top 10 DEmRNAs/DElncRNAs when comparing AS and normal controls. AS, ankylosing spondylitis; DEmRNA, differentially expressed mRNA; DElncRNA, differentially expressed long non-coding RNA.

HumanHT-12 V4.0 expression beadchip), which was downloaded from the Gene Expression Omnibus (GEO; www.ncbi. nlm.nih.gov/gds). The mRNA and lncRNA expression profile of 16 patients with AS and 16 normal controls (Australian cohort) in the GSE25101 dataset (GPL6947 Illumina HumanHT-12 V3.0 expression beadchip) was also downloaded from the GEO database. The expression levels of the selected DEmRNAs and DElncRNAs between the patients with AS and normal controls in the present study were validated using the GSE73754 and GSE25101 datasets, and the difference in the expression levels was visualized using box plots.

Receiver operating characteristic (ROC) analyses. To assess the diagnostic value of the DEmRNAs in AS, ROC analyses were conducted using pROC package (version 1.13.0) in $\mathrm{R}$ language (cran.r-project.org/web/packages/pROC/index.html). The area under the curve (AUC) under the binomial exact confidence interval was calculated. ROC curves were then generated.

Statistical analysis. Values are displayed as the mean \pm standard deviation. Student's t-test was used to assess differences among the groups, and $\mathrm{P} \leq 0.05$ was considered to indicate a statistically significant difference. Co-expression associations between the lncRNAs and the protein-coding genes were estimated using pairwise PCC analysis using R language (version 3.3.3; stat. ethz.ch/R-manual/R-devel/library/stats/html/hclust.html).

\section{Results}

Identification of DEmRNAs and DElncRNAs between patients with $A S$ and normal controls. Compared with the normal controls, 1,072 DEmRNAs (320 upregulated and 752 downregulated) and 372 DElncRNAs (230 upregulated and 142 downregulated) in patients with AS were identified. The top 10 up- and downregulated DEmRNAs and DElncRNAs between the patients with AS and normal controls are shown in Tables I and II, respectively.

MANSC domain containing 1 (MANSC1) and DNMT1 were the most significantly up- and downregulated DEmRNAs between the patients with AS and normal controls, respectively (data not shown). NOTCH1 associated lncRNA in T-cell acute lymphoblastic leukemia 1 (NALT1) and RP11-837J7.4 were the most significantly up- and downregulated DElncRNAs between the patients with AS and normal controls, respectively (data not shown).

AS-specific PPI network construction. The AS-specific PPI network consisted of 159 nodes and 164 edges. The top 10 mRNAs that had the highest degrees were Myc proto-oncogene basic helix-loop-helix transcription factor (MYC; degree=65), heterogeneous nuclear ribonucleoprotein A1 (HNRNPA1; degree=40), spectrin-a non-erythrocytic 1 (SPTAN1; degree=18), TATA-box binding protein associated factor 10 (TAF10; degree=9), ETS proto-oncogene 1 transcription factor (degree=7), NCK adaptor protein 1 (degree=6), eukaryotic translation initiation factor $4 \mathrm{~B}$ (degree=5), KIAA1033 (degree=5), extended synaptotagmin 1 (degree=5) and tumor protein 53 (degree=5); of these, MYC, HNRNPA1, SPTAN1 and TAF10 were hub proteins of the AS-specific PPI network (Fig. 1).

AS-specific weighted DEmRNA-DElncRNA co-expression network analysis. A total of 3,505 lncRNA-mRNA pairs were identified, which included 302 DElncRNAs and 602 DEmRNAs with IPCC value $\geq 0.90 \mathrm{l}$ and $\mathrm{P}<0.001$. Based on these IncRNA-mRNA pairs, the negatively and positively co-expressed DEmRNA-DElncRNA interaction networks were constructed, respectively. The top 100 co-expressed DEmRNA-DElncRNA interaction network is presented in Table III. Based on the positively co-expressed DEmRNA-DElncRNA interaction network, MSTRG.8559 (degree=226) and long intergenic 
A

Transcription, DNA-dependent

Signal transduction by p53-mediated apoptosis protein

Protein complex assembly

Negative regulation of transcription, DNA-dependent

Negative regulation of apoptotic process

Interspecies interaction between organisms

Gene expression

DNA repair

Chromatin modification

Cellular response to insulin stimulus

Cell surface receptor signaling pathway

Carbohydrate metabolic process

Blood coagulation

Base-excision repair
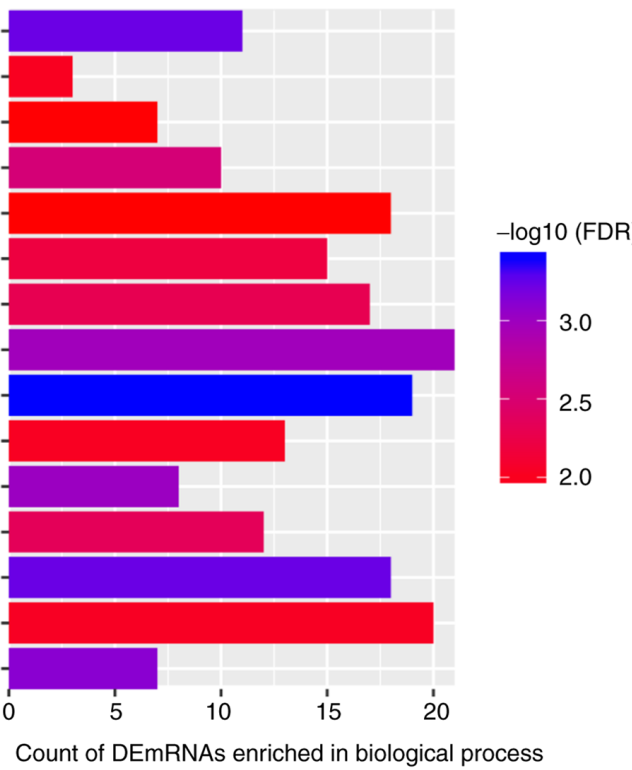

B Plasma membrane

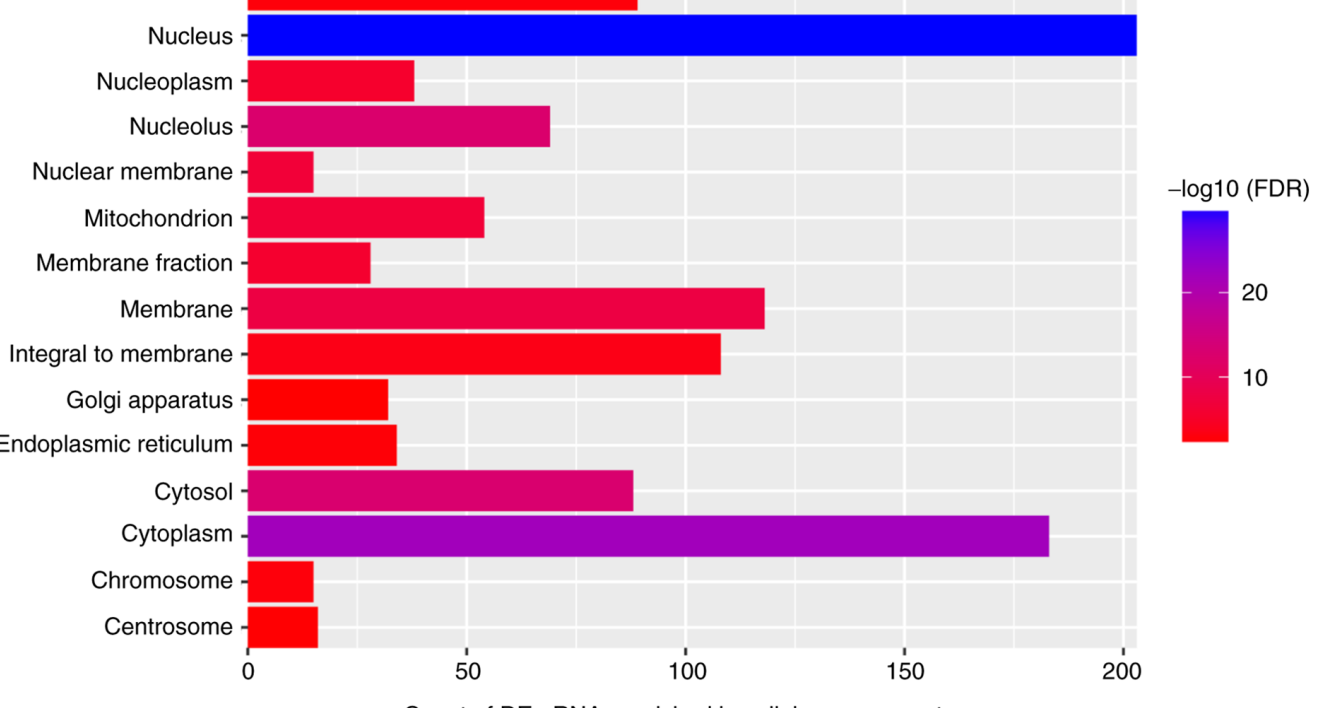

Count of DEmRNAs enriched in cellular component

C

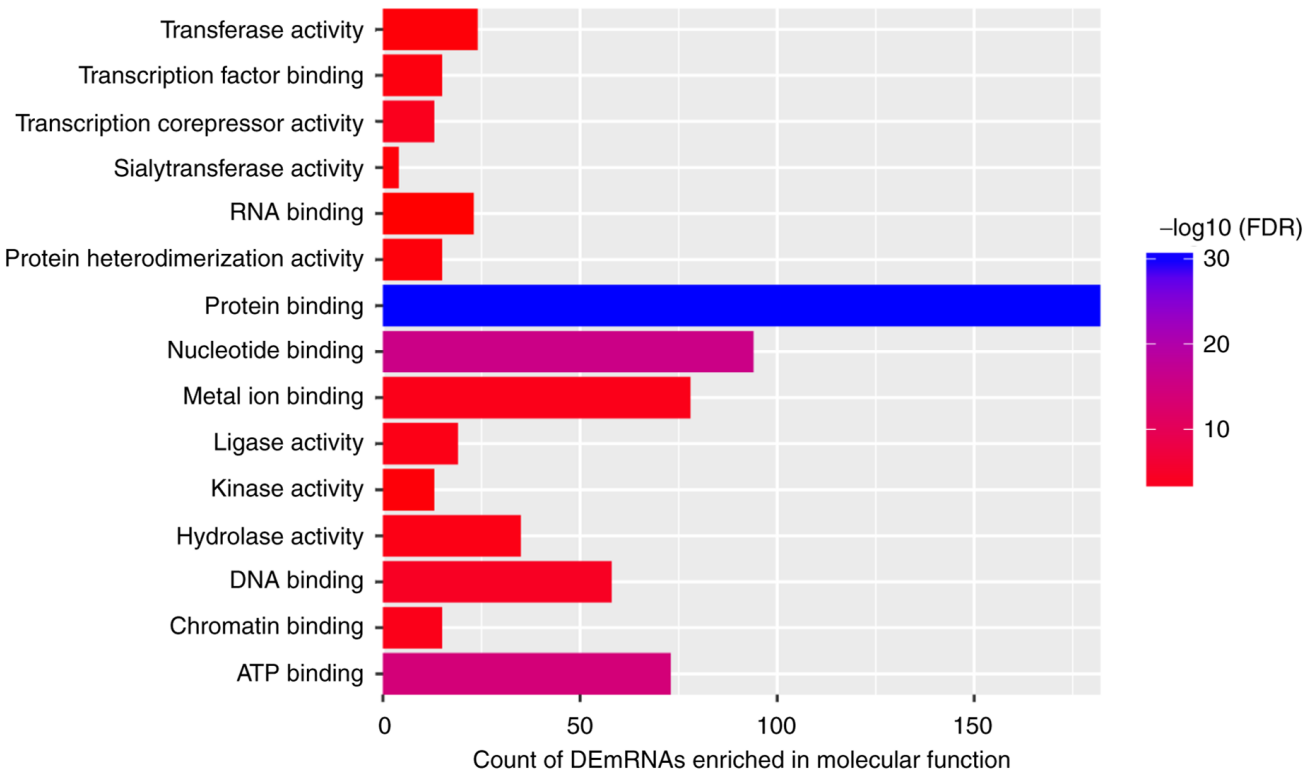

Figure 3. Top 15 most significantly enriched GO terms of DEmRNAs co-expressed with DElncRNAs. The $\mathrm{x}$-axis presents the number of DEmRNAs enriched in the GO terms and the y-axis presents the GO terms. (A) Biological process; (B) Cellular component; (C) Molecular function. GO, Gene Ontology; DEmRNA, differentially expressed mRNA; DElncRNA, differentially expressed long non-coding RNA; FDR, false discovery rate. 


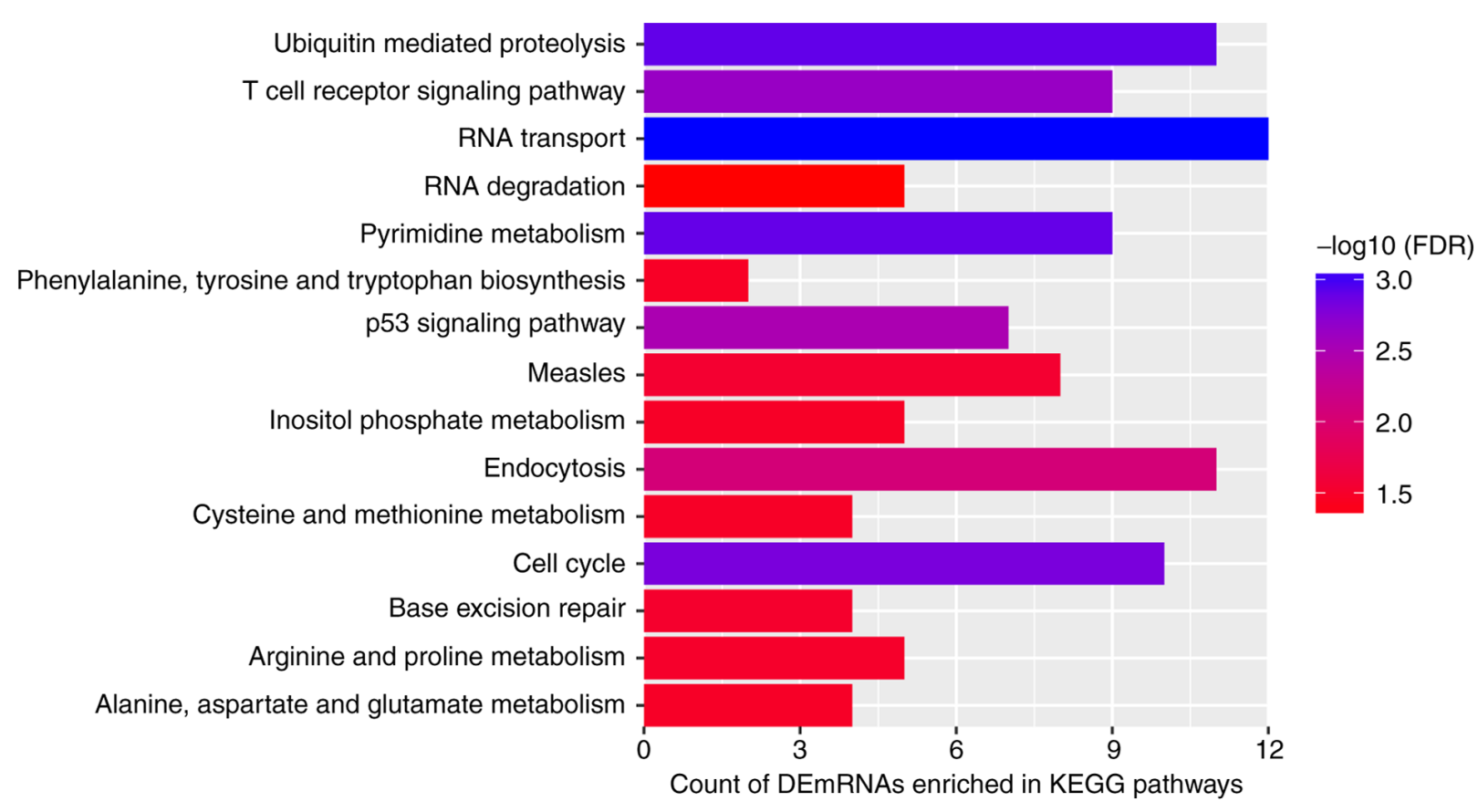

Figure 4. Top 15 most significantly enriched KEGG pathways of DEmRNAs co-expressed with DElncRNAs. The x-axis presents the number of DEmRNAs enriched in KEGG pathways and the y-axis presents the KEGG pathways. KEGG, Kyoto Encyclopedia of Genes and Genomes; DEmRNA, differentially expressed mRNA; DElncRNA, differentially expressed long non-coding RNA; FDR, false discovery rate.

non-protein coding RNA 987 (LINC00987; degree=209) were the top 2 DElncRNAs that were co-expressed with the greatest number of DEmRNAs (Fig. 2). The selected co-expression DEmRNA-DElncRNA interaction network is presented in Fig. 2.

Functional analysis of DEmRNAs co-expressing DElncRNAs between patients with AS and normal controls. Based on the GO enrichment analysis (Fig. 3) of the 602 DEmRNAs that were co-expressed with DElncRNAs in patients with AS and normal controls, the significantly enriched GO terms were as follows: 'DNA repair' (FDR $=3.94 \times 10^{-4}$; Fig. 3A), 'carbohydrate metabolic process'

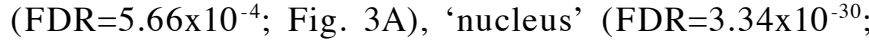

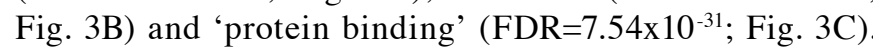
'T-cell receptor signaling pathway' (FDR $=2.29 \times 10^{-3}$; Fig. 4) and 'cell cycle' (FDR $=1.52 \times 10^{-3}$; Fig. 4) were the significantly enriched KEGG pathways.

DEmRNAs close to DElncRNAs with cis-regulatory effects. A total of 84 DElncRNAs and nearby cis target DEmRNA pairs, which included 73 DElncRNAs and 82 DEmRNAs, were obtained (Table IV).

Validation of the expression of DEmRNAs and DElncRNAs using the GSE73754 and GSE25101 datasets. A total of 4 DEmRNAs (DNMT1, PDCD1, CARD11 and PLCG1) were selected to perform expression validation using the GSE73754 dataset (Fig. 5). The expression of all four DElncRNAs was downregulated in patients with AS when compared with the normal controls, which was generally consistent with the RNA sequencing data (DNMT1, $\mathrm{P}<0.05$; CARD11, $\mathrm{P}<0.05$; PDCD1, P>0.05; PLCG1, P>0.05). Three lncRNAs (cat eye syndrome chromosome region candidate 7 , hydatidiform mole associated and imprinted and KIAA0125) and seven DEmRNAs [MANSC1, adenosine triphosphatase sarcoplasmic/endoplasmic reticulum $\mathrm{Ca}^{2+}$ transporting 2 (ATP2A2), myeloid zinc finger 1 (MZF1), PDCD1, DNMT1, CARD11 and PLCG1] were selected to perform expression validation using the GSE25101 dataset (Fig. 6). The expression of KIAA0125 $(\mathrm{P}>0.05)$, MANSC1 $(\mathrm{P}>0.05)$, ATP2A2 $(\mathrm{P}>0.05)$, MZF1 $(\mathrm{P}>0.05)$, PDCD1 $(\mathrm{P}>0.05)$, DNMT1 $(\mathrm{P}<0.0001)$, CARD11 $(\mathrm{P}<0.05)$ and PLCG1 $(\mathrm{P}>0.05)$ was generally consistent with the RNA sequencing data.

ROC curve analysis. ROC curve analyses and the AUC were used to assess the discriminatory ability of the four DEmRNAs (DNMT1, PDCD1, CARD11 and PLCG1) among the 52 patients with AS and 20 normal controls of the GSE73754 dataset. The AUCs of PDCD1 and PLCG1 were $<0.7$ (data not shown). The AUCs of CARD11 and DNMT1 were 0.782 and 0.737 , respectively (Fig. 7). For AS diagnosis, the sensitivity (proportion of true positive) and 1-specificity (proportion of false positive) of CARD11 were 70.0 and $80.8 \%$, respectively, and for DNMT1 were 75.0 and 69.2\%, respectively (Fig. 7).

\section{Discussion}

AS is a type of autoimmune disorder that is associated with HLB-27 and T-cells; however, its etiology and pathogenesis remain unclear (18). The delays in the diagnosis of AS and the insufficient responses to the currently available therapeutics supports the requirement for a greater understanding of its pathogenesis.

A previous microarray study identified four lncRNAs, lnc-ZNF354A-1, lnc-LIN54-1, lnc-FRG2C-3 and lnc-USP50-2, that are involved in the abnormal osteogenic differentiation 
Table IV. Nearby targeted DEmRNAs of DElncRNAs between ankylosing spondylitis and normal controls.

\begin{tabular}{|c|c|c|c|c|c|c|}
\hline \multirow[b]{2}{*}{ Chr } & \multicolumn{3}{|c|}{ DElncRNA } & \multicolumn{3}{|c|}{ Nearby DEmRNA } \\
\hline & Symbol & Start $-100 \mathrm{~kb}$ & End $+100 \mathrm{~kb}$ & Symbol & Start & End \\
\hline $\operatorname{chr} 1$ & PIK3CD-AS1 & $9,552,610$ & $9,754,586$ & CLSTN1 & $9,729,026$ & 9824526 \\
\hline chr14 & LINC01588 & $49,827,571$ & $50,192,643$ & SOS2 & $50,117,120$ & $50,231,558$ \\
\hline $\operatorname{chr} 22$ & AC004471.9 & $19,021,529$ & $19,224,503$ & SLC25A1 & $19,175,575$ & $19,178,830$ \\
\hline chr2 & AC007879.5 & $207,139,864$ & $207,629,795$ & KLF7 & $207,074,137$ & $207,167,267$ \\
\hline ChrX & INE1 & $47,104,921$ & $47,305,865$ & UBA1 & $47,190,861$ & $472,151,28$ \\
\hline ChrX & INE1 & $47,104,921$ & $47,305,865$ & USP11 & $47,232,690$ & $47,248,328$ \\
\hline chr1 & TAF1A-AS1 & $222,489,825$ & $222,693,032$ & MIA3 & $222,618,086$ & $222,668,012$ \\
\hline $\operatorname{chr} 7$ & AC007285.6 & $29,888,600$ & $30,125,660$ & SCRN1 & $29,920,103$ & $29,990,289$ \\
\hline chr1 & RP11-318C24.2 & $175,804,762$ & $176,020,513$ & RFWD2 & $175,944,831$ & $176,207,493$ \\
\hline $\operatorname{chr} 5$ & AC008781.7 & $141,518,414$ & $141,726,481$ & DIAPH1 & $141,515,016$ & $141,619,055$ \\
\hline $\operatorname{chr} 3$ & U73166.2 & $50,160,303$ & $50,363,358$ & HYAL3 & $50,292,831$ & $50,299,468$ \\
\hline ChrX & RP13-314C10.5 & $23,672,992$ & $23,882,956$ & PRDX4 & $23,664,262$ & $23,686,399$ \\
\hline $\operatorname{chr} 1$ & RP11-195C7.1 & $176,107,648$ & $176,329,330$ & RFWD2 & $175,944,831$ & $176,207,493$ \\
\hline $\operatorname{chr} 21$ & AP001056.1 & $44,075,489$ & $44,276,453$ & PWP2 & $44,107,290$ & $44,131,181$ \\
\hline $\operatorname{chr} 21$ & AP001056.1 & $44,075,489$ & $44,276,453$ & TRAPPC10 & $44,012,319$ & $44,106,552$ \\
\hline $\operatorname{chr} 1$ & RP11-7O11.3 & $43,844,370$ & $44,046,551$ & ST3GAL3 & $43,705,824$ & $43,931,165$ \\
\hline $\operatorname{chr} 3$ & RP11-379K17.4 & $169,839,353$ & $170,066,734$ & GPR160 & $170,037,929$ & $170,085,403$ \\
\hline $\operatorname{chr} 2$ & AC092620.2 & 138469090 & $138,674,458$ & SPOPL & $138,501,801$ & $138,573,547$ \\
\hline $\operatorname{chr} 3$ & LINC00969 & $195,558,062$ & $195,839,964$ & MUC20 & $195,720,882$ & $195,741,123$ \\
\hline $\operatorname{chr} 4$ & RP11-15B17.1 & $99,850,006$ & $100,295,099$ & LAMTOR3 & $99,878,336$ & $99,894,490$ \\
\hline $\operatorname{chr} 5$ & SCAMP1-AS1 & $78,242,365$ & $78,460,507$ & AP3B1 & $78,000,525$ & $78,294,755$ \\
\hline $\operatorname{chr} 15$ & RP11-30K9.6 & $58,668,072$ & $58,870,974$ & ADAM10 & $58,588,807$ & $58,749,978$ \\
\hline chr8 & BAALC-AS1 & $103,056,990$ & $103,398,772$ & SLC25A32 & $103,39,8635$ & $103,415,189$ \\
\hline $\operatorname{chr} 5$ & RP11-159F24.5 & $43,415,274$ & $43,625,310$ & NNT & $43,602,692$ & $43,707,405$ \\
\hline $\operatorname{chr} 5$ & LINC01187 & $170,091,579$ & $170,299,141$ & FOXI1 & $17,010,5897$ & $170,109,725$ \\
\hline chr8 & LINC01151 & $122,570,385$ & $122,794,106$ & ZHX2 & $122,781,394$ & $122,974,512$ \\
\hline $\operatorname{chr11}$ & RP11-727A23.4 & $83,080,144$ & $83,284,520$ & PCF11 & $83,156,988$ & $83,187,451$ \\
\hline chr8 & GS1-393G12.12 & $144,214,590$ & $144,415,138$ & BOP1 & $144,262,102$ & $144,291,370$ \\
\hline $\operatorname{chr} 12$ & RP11-561P12.5 & $8,448,361$ & $8,667,613$ & CLEC4E & $8,533,305$ & $8,540,963$ \\
\hline $\operatorname{chr} 12$ & RP11-561P12.5 & $8,448,361$ & $8,667,613$ & CLEC4D & $8,509,475$ & $8,522,366$ \\
\hline $\operatorname{chr} 12$ & RP11-996F15.2 & $29,180,418$ & $29,417,848$ & ERGIC2 & $29,337,352$ & $29,381,189$ \\
\hline chr14 & RP11-44N21.1 & $104,993,609$ & $105,199,004$ & CDCA4 & $105,009,573$ & $105,021,148$ \\
\hline $\operatorname{chr} 14$ & RP11-44N21.1 & $104,993,609$ & $105,199,004$ & C14 or $\mathrm{f} 79$ & $104,985,775$ & $105,010,482$ \\
\hline $\operatorname{chr} 12$ & RP11-753H16.3 & $54,253,661$ & $54,597,688$ & HNRNPA1 & $54,280,193$ & $54,287,088$ \\
\hline chr14 & RP11-193F5.1 & $60,779,714$ & $61,082,585$ & SLC38A6 & $60,981,114$ & $61,083,733$ \\
\hline chr14 & RP5-1021I20.1 & $73,687,360$ & $73,903,270$ & PNMA1 & $73,711,783$ & $73,714,372$ \\
\hline $\operatorname{chr} 3$ & AC099668.5 & $49,584,480$ & $49,784,983$ & MST1 & $49,683,947$ & $49,689,501$ \\
\hline chr6 & RP11-425D10.10 & $109,282,795$ & $109,483,666$ & SMPD2 & $109,440,763$ & $109,443,919$ \\
\hline $\operatorname{chr} 14$ & CTD-2547L24.4 & $91,158,299$ & $91,359,003$ & CCDC88C & $91,271,323$ & $91,417,844$ \\
\hline chr14 & RP11-524O1.4 & $21,284,292$ & $21,484,920$ & CHD8 & $21,385,194$ & $21,456,126$ \\
\hline chr14 & RP11-524O1.4 & $21,284,292$ & $21,484,920$ & SUPT16H & $21,351,472$ & $21,384,266$ \\
\hline chr16 & RP11-459F6.3 & $58,029,529$ & $58,259,133$ & CFAP20 & $58,113,588$ & $58,129,450$ \\
\hline chr16 & RP11-264B17.3 & $28,874,804$ & $29,090,775$ & LAT & $28,984,826$ & $28,990,783$ \\
\hline chr1 & RP11-196G18.22 & $149,744,498$ & $149,949,024$ & HIST2H2BF & $149,782,689$ & $149,812,373$ \\
\hline $\operatorname{chr} 16$ & LA16c-306E5.3 & $3,358,071$ & $3,615,564$ & NLRC3 & $3,539,033$ & $3,577,400$ \\
\hline chr16 & RP11-461A8.4 & $3,550,636$ & $3,751,703$ & NLRC3 & $3,539,033$ & $3,577,400$ \\
\hline $\operatorname{chr} 17$ & RP1-59D14.5 & $2,275,061$ & $2,479,306$ & TSR1 & $2,322,503$ & $2,337,507$ \\
\hline $\operatorname{chr} 17$ & RP1-59D14.5 & $2,275,061$ & $2,479,306$ & METTL16 & $2,405,562$ & $2,511,891$ \\
\hline $\operatorname{chr} 17$ & RP1-59D14.5 & $2,275,061$ & $2,479,306$ & SMG6 & $2,059,839$ & $2,303,771$ \\
\hline $\operatorname{chr} 17$ & RP11-927P21.2 & $64,799,766$ & $65,000,716$ & LRRC37A3 & $64,854,312$ & $64,919,480$ \\
\hline
\end{tabular}


Table IV. Continued.

\begin{tabular}{|c|c|c|c|c|c|c|}
\hline \multirow[b]{2}{*}{$\mathrm{Chr}$} & \multicolumn{3}{|c|}{ DElncRNA } & \multicolumn{3}{|c|}{ Nearby DEmRNA } \\
\hline & Symbol & Start $-100 \mathrm{~kb}$ & End $+100 \mathrm{~kb}$ & Symbol & Start & End \\
\hline $\operatorname{chr} 17$ & RP11-1094M14.11 & $35,468,109$ & $35,674,843$ & SLFN14 & $35,548,125$ & $35,558,098$ \\
\hline $\operatorname{chr17}$ & CTD-2534I21.8 & $44,847,912$ & $45,048,939$ & EFTUD2 & $44,849,943$ & $44,899,662$ \\
\hline chr19 & PTOV1-AS1 & $49,738,639$ & $49,951,676$ & NUP62 & $49,906,825$ & $49,929,763$ \\
\hline chr20 & RP4-591C20.9 & $63,761,212$ & $63,964,293$ & UCKL1 & $63,939,829$ & $63,956,415$ \\
\hline chr19 & AC007292.3 & $4,256,637$ & $4,458,448$ & CCDC94 & $4,247,079$ & $4,269,090$ \\
\hline chr19 & RAB11B-AS1 & $8,274,373$ & $8,490,685$ & RAB11B & $8,389,981$ & $8,404,434$ \\
\hline chr5 & CTD-2033C11.1 & $65,824,629$ & $66,025,135$ & ERBIN & $65,926,475$ & $66,082,549$ \\
\hline chr4 & RP11-572O17.1 & $1,612,821$ & $1,813,622$ & FAM53A & $1,617,915$ & $1,684,302$ \\
\hline chr4 & RP11-572O17.1 & $1,612,821$ & $1,813,622$ & LETM1 & $1,811,479$ & $1,856,247$ \\
\hline chr1 & RP4-736L20.3 & $10,329,881$ & $10,530,677$ & DFFA & $10,456,522$ & $10,472,526$ \\
\hline $\operatorname{chr} 10$ & RP11-574K11.29 & $73,603,735$ & $73,813,581$ & USP54 & $73,497,538$ & $73,625,953$ \\
\hline chr1 & RP11-156E8.1 & $244,869,350$ & $245,071,088$ & EFCAB2 & $244,969,705$ & $245,127,164$ \\
\hline chr3 & RP11-767C1.2 & $12,732,219$ & $12,932,728$ & IQSEC1 & $12,897,220$ & $13,073,117$ \\
\hline chr1 & RP11-11N7.4 & $244,764,738$ & $244,965,272$ & HNRNPU & $244,840,638$ & $244,864,560$ \\
\hline chr3 & RP11-778D9.13 & $184,032,942$ & $184,233,561$ & AP2M1 & $184,174,689$ & $184,184,091$ \\
\hline $\operatorname{chr} 12$ & RP1-197B17.4 & $47,631,908$ & $47,832,351$ & HDAC7 & $47,782,722$ & $47,833,132$ \\
\hline $\operatorname{chr} 17$ & AC142472.6 & $45,046,730$ & $45,248,470$ & NMT1 & $45,051,610$ & $45,109,016$ \\
\hline $\operatorname{chr} 15$ & CTD-2562G15.3 & $75,352,964$ & $75,553,947$ & SIN3A & $75,369,379$ & $75,455,842$ \\
\hline chr17 & RP11-333J10.3 & $36,898,598$ & $37,100,034$ & AATF & $36,948,875$ & $37,056,871$ \\
\hline chr15 & CTD-2382E5.6 & $41,808,204$ & $42,008,714$ & JMJD7 & $41,828,085$ & $41,837,581$ \\
\hline $\operatorname{chr} 18$ & RP11-405M12.3 & $74,970,197$ & $75,171,091$ & ZNF407 & $74,597,870$ & $75,065,671$ \\
\hline $\operatorname{chr} 17$ & HEXDC-IT1 & $82,325,498$ & $82,527,310$ & NARF & $82,458,180$ & $82,490,537$ \\
\hline $\operatorname{chr} 17$ & RP11-159D12.6 & $57,906,674$ & $58,108,187$ & CUEDC1 & $57,861,243$ & $57,955,323$ \\
\hline $\operatorname{chr} 15$ & RP11-1H8.5 & 34315,450 & $34,520,273$ & LPCAT4 & $34,358,618$ & $34,367,278$ \\
\hline chr15 & RP11-1H8.5 & $34,315,450$ & $34,520,273$ & SLC12A6 & $34,229,996$ & $34,338,060$ \\
\hline chr5 & CTC-487M23.6 & $112,794,933$ & $112,996,531$ & SRP19 & $112,861,222$ & $112,869,788$ \\
\hline chr5 & CTC-487M23.6 & $112,794,933$ & $112,996,531$ & DCP2 & $112,976,702$ & $113,020,970$ \\
\hline chr5 & AC005593.2 & $131,697,415$ & $131,897,929$ & FNIP1 & $131,641,714$ & $131,797,063$ \\
\hline $\operatorname{chr} 17$ & RP13-638C3.5 & $82,548,849$ & $82,750,657$ & FN3KRP & $82,716,683$ & $82,730,328$ \\
\hline chr14 & RP11-298I3.6 & $22,923,083$ & $23,124,217$ & AJUBA & $22,971,174$ & $22,982,642$ \\
\hline $\operatorname{chr} 16$ & RP11-451N19.3 & $58,605,799$ & $58,806,297$ & SLC38A7 & $58,665,109$ & $58,685,104$ \\
\hline chr16 & RP11-451N19.3 & $58,605,799$ & $58,806,297$ & GOT2 & $58,707,131$ & $58,734,357$ \\
\hline chr19 & CTD-2233K9.1 & $46,846,535$ & $47,049,156$ & ARHGAP35 & $46,918,676$ & $47,005,077$ \\
\hline chr6 & XXbac-BPG283O16.9 & $30,182,349$ & $30,386,054$ & RPP21 & $30,345,131$ & $30,346,884$ \\
\hline chr6 & XXbac-BPG283O16.9 & $30,182,349$ & $30,386,054$ & TRIM26 & $30,184,455$ & $30,213,427$ \\
\hline
\end{tabular}

Chr, chromosome; DEmRNAs, differentially expressed mRNAs; DElncRNA, differentially expressed long non-coding RNA.

of mesenchymal stem cells (MSCs) in patients with AS. The expression of these four lncRNAs was positively correlated with that of bone morphogenetic protein 2 and Noggin in MSCs from healthy donors (8). A recent study reported that IncRNA-AK001085 was downregulated in patients with AS and served as a potential diagnostic indicator, thus, IncRNA-AK001085 was considered to be a potential suppressor of AS (9). Due to the lack of research, the regulatory mechanism of the majority of lncRNAs in AS remains unknown. In the present study, the key DEmRNAs and DElncRNAs associated with AS were identified and their functions in AS were investigated using RNA sequencing and bioinformatics analysis.

LINC00342 was demonstrated to be upregulated in patients with non-small cell lung cancer and its expression was positively correlated with lymph node metastasis and the Tumor-Node-Metastasis stage (19). Coiled-coil domain containing 26 (CCDC26) is also a tumor-associated lncRNA that regulates the growth of glioma, pancreatic cancer and myeloid leukemia cells (20-22). In the present study, LINC00342 and CCDC26 were downregulated in patients with AS, which suggests that they may serve roles in AS. 
A

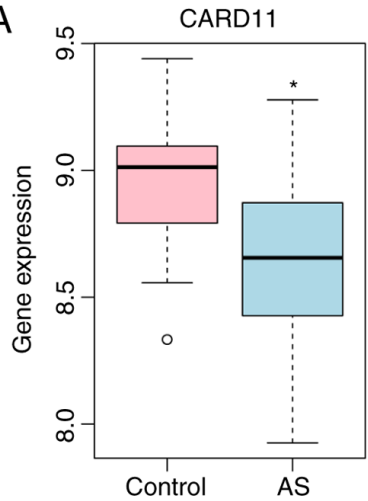

B

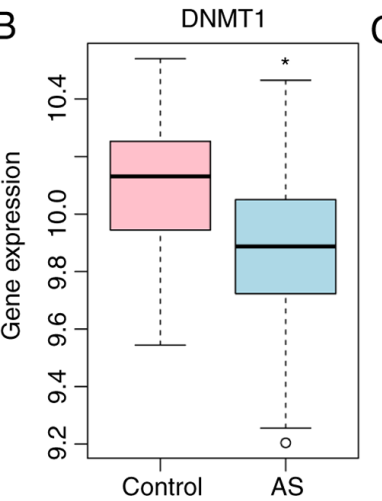

C

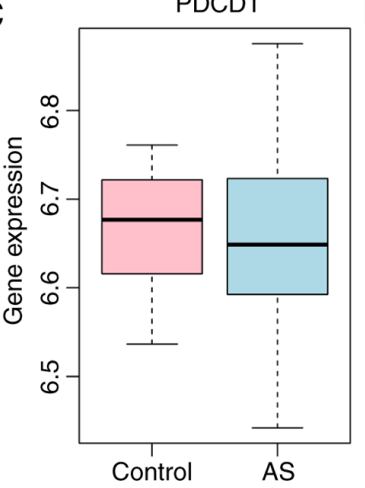

D

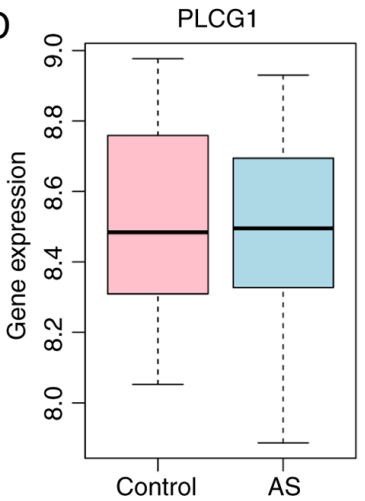

Figure 5. Validation the expression of selected DEmRNAs between patients with AS and normal controls in the GSE73754 dataset. The x-axis presents the AS and normal control groups and the y-axis presents the expression levels of (A) CARD11, (B) DNMT1, (C) PDCD1 and (D) PLCG1. Data are presented as the median and the 75 th and 25 th percentiles. ${ }^{*} \mathrm{P}<0.05$ vs. control. AS, ankylosing spondylitis; DEmRNA, differentially expressed mRNA; CARD11, caspase recruitment domain-containing protein 11; DNMT1, DNA methyltransferase 1; PDCD1, programmed cell death 1; PLCG1, phospholipase C $\gamma 1$.

A

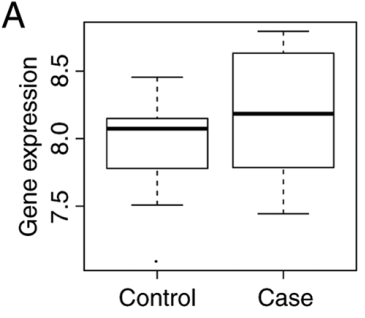

MANSC1

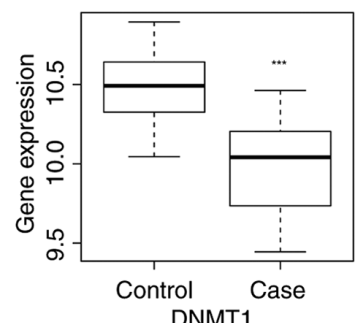

DNMT1

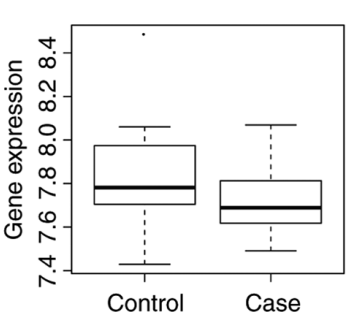

ATP2A2

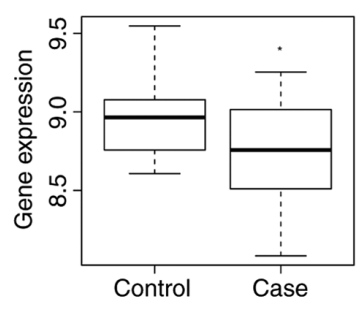

CARD11

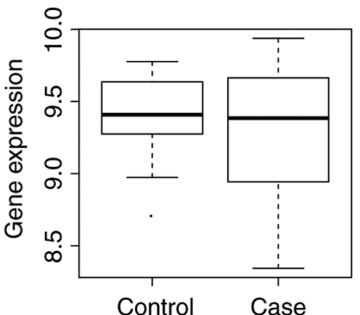

MZF1

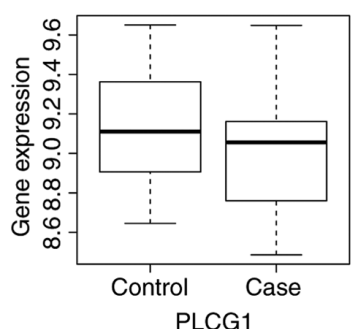

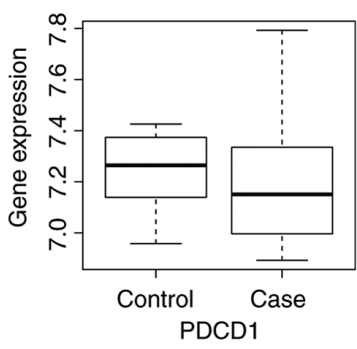

PDCD1
B

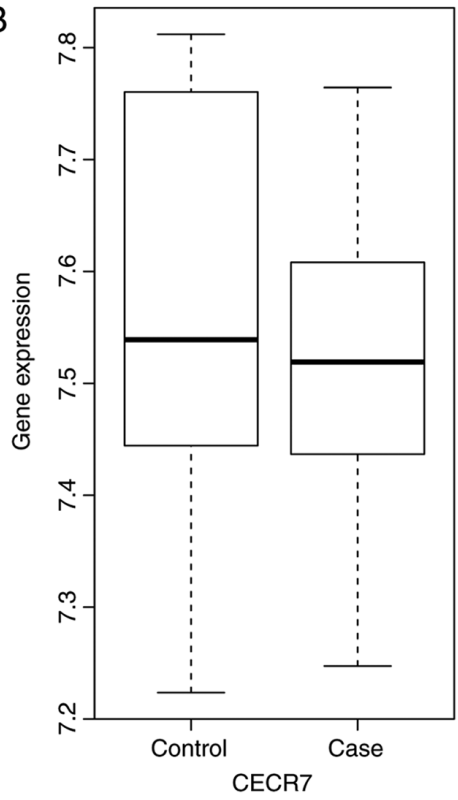

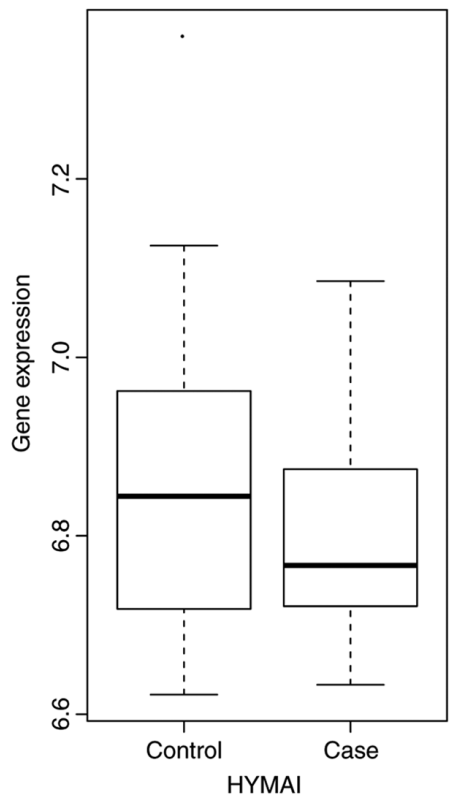

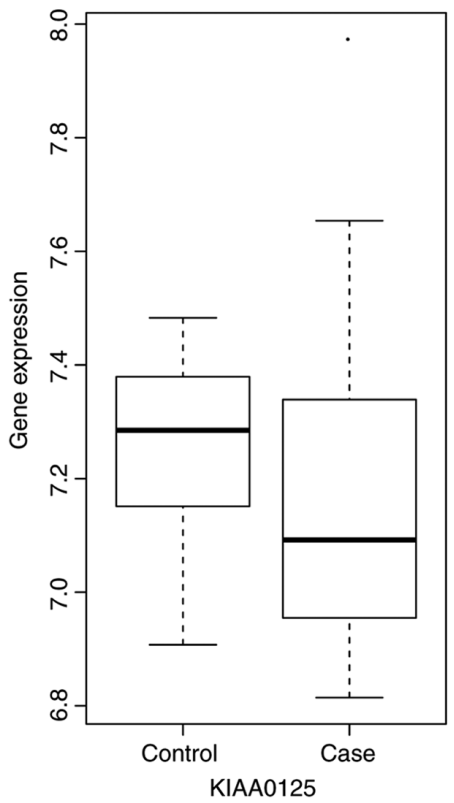

Figure 6. Validation of the expression of selected DEmRNAs and DElncRNAs between patients with AS and the normal controls in the GSE25101 dataset. The $\mathrm{X}$-axis presents the AS and normal control groups and the $\mathrm{y}$-axis presents the expression levels. (A) The selected DEmRNAs and (B) The selected DElncRNAs. AS, ankylosing spondylitis; DEmRNA, differentially expressed mRNA; DElncRNA, differentially expressed long non-coding RNA; CARD11, caspase recruitment domain-containing protein 11; DNMT1, DNA methyltransferase 1; PDCD1, programmed cell death 1; PLCG1, phospholipase C $\gamma 1$; MANSC1, MANSC domain containing 1; ATP2A2, adenosine triphosphatase sarcoplasmic/endoplasmic reticulum Ca ${ }^{2+}$ transporting 2; MZF1, myeloid zinc finger 1; CECR7, cat eye syndrome chromosome region candidate 7; HYMAI, hydatidiform mole associated and imprinted. 
A

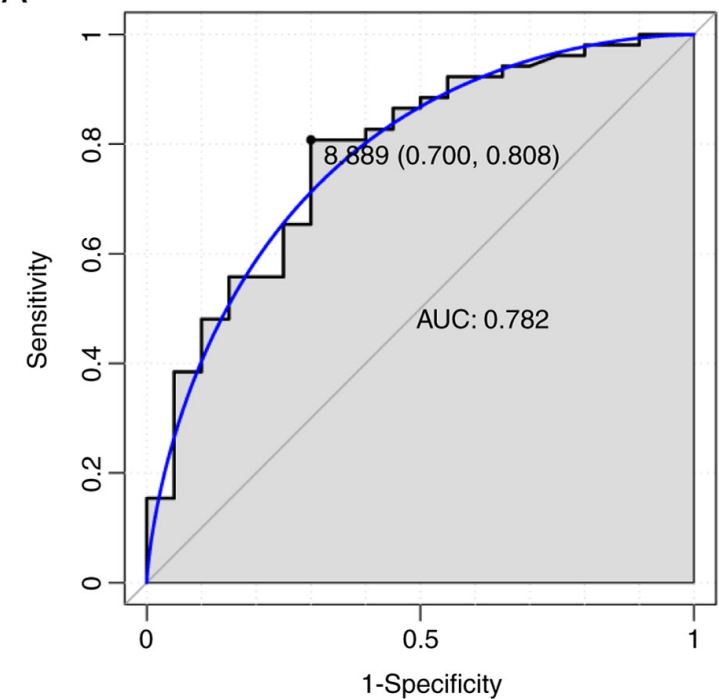

B

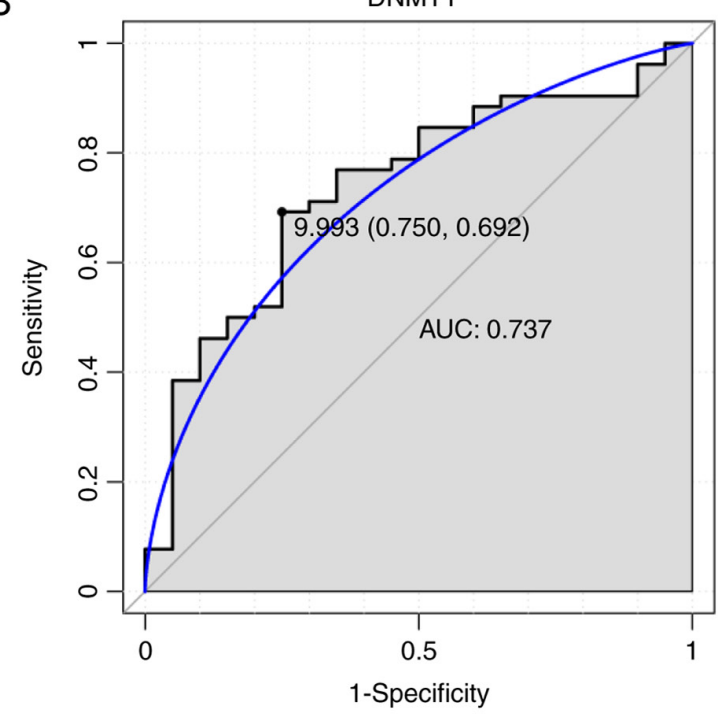

Figure 7. ROC curves of CARD11 and DNMT1 comparing patients with ankylosing spondylitis and normal controls in the GSE73754 dataset. ROC curves displayed the diagnostic ability of differentially expressed long non-coding RNA with sensitivity (the proportion of true positive) and 1-Specificity (the proportion of false positive). The $\mathrm{x}$-axis presents 1-specificity and y-axis presents sensitivity. (A) CARD11 and (B) DNMT1. ROC, receiver operating characteristic; CARD11, caspase recruitment domain-containing protein 11; DNMT1, DNA methyltransferase 1; AUC, area under the curve.

Further studies are required in order to identify their precise function in AS.

Furthermore, the results of the present study indicated that many novelDElncRNAs may be involved in AS.In ordertoinvestigate their functions in AS, a weighted DElncRNA-DEmRNA co-expression network was constructed and functional annotation of the DElncRNAs co-expressed with DElncRNAs was performed. A total of 3,505 DElncRNA-DEmRNA co-expression pairs, which included 302 DElncRNAs and 602 DEmRNAs, were obtained. Based on the DEmRNAs co-expressed with DElncRNAs, 'T-cell receptor signaling pathway' was a significantly enriched pathway. T-cells have been demonstrated by previous studies to serve important roles in the pathology of AS (23-25). HLA-B27-reactive cluster of differentiation- $4^{+}$T-cells have been reported to be involved in the pathogenesis of spondyloarthropathies (26). The number of peripheral T-helper (Th)-2 and Th17 lymphocytes has been demonstrated to be increased in AS, which is suggestive of their potential roles in AS $(27,28)$. Therefore, the results of the present study support those of previous studies as well as the importance of the T-cell receptor signaling pathway in AS. Furthermore, the DEmRNAs that were enriched in the $\mathrm{T}$-cell receptor signaling pathway, including NF- $\kappa \mathrm{B}$ inhibitor $\beta$, CARD11, P21 (Ras-related C3 botulinum toxin substrate 1) activated kinase 6 , protein kinase $\mathrm{C} \theta$, PLCG1, PDCD1, FYN proto-oncogene Src family tyrosine kinase, protein kinase B2 and Vav guanine nucleotide exchange factor 3, may be involved with AS by regulating the T-cell receptor signaling pathway.

Among these DEmRNAs, PDCD1 is a known AS-associated gene. PDCD1 is a member of the immunoglobulin superfamily that is expressed on the surface of peripheral T-cells, and regulates T-cell responses and the maintenance of peripheral tolerance $(29,30)$. A previous study demonstrated that the expression levels of PDCD1 on activated T-cells were decreased in patients with AS (1). Downregulation of PDCD1 may be involved in AS by stimulating the activity of T-cells and elevating the produc- tion of cytokines, which promotes spinal inflammation and destruction in patients with AS (1). Downregulated PDCD1 was also identified in patients with AS in the present study, which provides evidence to support the results of the previous study.

CARD11 is a shared member of the CARD and CARD-containing membrane-associated guanylate kinase protein 1 families that has been reported to serve a vital role in regulation of inflammation and the immune response (31). Although to the best of our knowledge, there have been no studies that have reported on the association between CARD11 and AS, downregulated CARD11 has been implicated in another type of autoimmune disease, rheumatoid arthritis, via $\mathrm{NF}-\kappa \mathrm{B}$ activation, reduced Th17 responses and the decreased production of proinflammatory cytokines, including IL-1 $\beta$, IL-6 and IL-17 (10,32,33). Joint inflammation and destruction were demonstrated to be attenuated by CARD11 small interfering RNA treatment in mice (10). Considering the crucial roles of NF- $\kappa \mathrm{B}$ activation, Th17 cells and proinflammatory cytokines in AS, it was hypothesized that CARD11 may also be a key regulator of AS.

Similar to CARD11, PLCG1 has been reported to be associated with other types of autoimmune disease, including multiple sclerosis and lymphoproliferative syndrome $(11,12)$. Furthermore, the interaction between PLCG1 and linker for activation of T-cells (LAT) was revealed to be involved with the activation and proliferation of T-cells $(11,12)$. The production of IL- 6 by T-cells is also regulated by PLCG1-LAT $(11,12)$. Therefore, downregulated PLCG1 in the patients with AS in the present study may serve important roles in the progression of AS by regulating T-cells and the production of IL- 6 .

DNMT1 encodes an enzyme that establishes and regulates patterns of methylated cytosine residues (13). A previous study observed downregulated and hypermethylated DNMT1 in the peripheral blood mononuclear cells of patients with AS when compared with normal controls, which suggests that DNMT1 may be a potential biomarker of AS (13). Thus, the downregu- 
lation of DNMT1 observed in patients with AS in the present study is consistent with this previous study.

Based on the ROC analysis in the present study, CARD11 and DNMT1 may have great diagnostic value for AS, and therefore may be potential biomarkers.

LINC00987 was a downregulated lncRNA in the patients with AS in the present study, and was co-expressed with DNMT1, CARD11 and PLCG1. In addition, DNMT1 and PLCG1 were co-expressed with another downregulated IncRNA, MSTRG.8559, in the patients with AS. Furthermore, LINC00987 and MSTRG.8559 were two hub lncRNAs of the positively co-expressed DElncRNA-DEmRNA network, which regulates the majority of the DEmRNAs in AS. It was hypothesized that these two DElncRNAs may serve crucial roles by regulating the expression of DNMT1, the T-cell receptor signaling pathway and its associated genes. Further studies are required to further investigate the biological functions of LINC00987 and MSTRG.8559, particularly those in AS.

RP11-837J7.4 and NALT1 were the most significantly down- and upregulated, respectively, DElncRNAs in patients with AS in the present study; however, their biological functions remain known. Further studies are required in order to identify whether these two DElncRNAs could serve as diagnostic biomarkers for AS.

Previous studies have revealed the prevalence of lncRNA-mediated cis regulation on nearby transcription (34-36). The 84 AS-specific DElncRNA and nearby cis target DEmRNA pairs obtained in the present study provide novel information for understanding the biological functions of lncRNAs in AS.

In conclusion, the present study obtained lncRNA and mRNA expression profiles from patients with AS and normal controls using RNA sequencing. A number of key genes, including PDCD1, DNMT1, CARD11 and PLCG1, that are involved in AS were identified. In addition, the results indicated that numerous novel DElncRNAs may be involved in AS. Furthermore, the functions of DElncRNAs in AS were investigated using functional annotation of DEmRNAs co-expressed with DElncRNAs and through the identification of nearby target DEmRNAs of DElncRNAs. These results may support further studies on the potential roles of lncRNAs in AS. However, the sample size for RNA-seq was small, which is a limitation of the present study, therefore, studies with larger sample sizes are required in order to confirm this conclusion.

\section{Acknowledgements}

The authors would like to thank Beijing Yangshen Bioinformatic Technology (Beijing, China) for their assistance during high-throughput sequencing and data analysis.

\section{Funding}

No funding was received.

\section{Availability of data and materials}

The datasets used and/or analyzed during the current study are available from the corresponding author on reasonable request.

\section{Authors' contributions}

ZX and GC conceived and designed the experiments. ZX and HL performed the experiments. ZX and QC analyzed the data. HL and QC were significant contributors in the manuscript. All authors revised the manuscript and have agreed to the publication of this manuscript.

\section{Ethics approval and consent to participate}

All of the participants submitted written informed consent and the present study was approved by the Ethics Committee of the 2nd Affiliated Hospital, School of Medicine, Zhejiang University (Zhejiang, China).

\section{Patient consent for publication}

Not applicable.

\section{Competing interests}

The authors declare that they have no competing interests.

\section{References}

1. Zhou L, Zhang Y, Xu H, Hu L, Zhang C, Sun L, Xie Y, Lu H, Zhang Z, Hu W and Lin X: Decreased programmed death-1 expression on the $\mathrm{T}$ cells of patients with ankylosing spondylitis. Am J Med Sci 349: 488-492, 2015.

2. Assassi S, Reveille JD, Arnett FC, Weisman MH, Ward MM, Agarwal SK, Gourh P, Bhula J, Sharif R, Sampat K, et al: Whole-blood gene expression profiling in ankylosing spondylitis shows upregulation of toll-like receptor 4 and 5. J Rheumatol 38: 87-98, 2011.

3. El Maghraoui A: Extra-articular manifestations of ankylosing spondylitis: Prevalence, characteristics and therapeutic implications. Eur J Intern Med 22: 554-560, 2011.

4. Smith JA: Update on ankylosing spondylitis: Current concepts in pathogenesis. Curr Allergy Asthma Rep 15: 489, 2015.

5. Evans DM, Spencer CC, Pointon JJ, Su Z, Harvey D, Kochan G, Oppermann U, Dilthey A, Pirinen M, Stone MA, et al: Interaction between ERAP1 and HLA-B27 in ankylosing spondylitis implicates peptide handling in the mechanism for HLA-B27 in disease susceptibility. Nat Genet 43: 761-767, 2011.

6. Davidson SI, Liu Y, Danoy PA, Wu X, Thomas GP, Jiang L, Sun L, Wang N, Han J, Han H, et al: Association of STAT3 and TNFRSF1A with ankylosing spondylitis in han chinese. Ann Rheum Dis 70: 289-292, 2011.

7. Wright MW and Bruford EA: Naming 'junk': Human non-protein coding RNA (ncRNA) gene nomenclature. Hum Genomics 5: 90-98, 2011.

8. Xie Z, Li J, Wang P, Li Y, Wu X, Wang S, Su H, Deng W, Liu Z, Cen $S$, et al: Differential expression profiles of long noncoding RNA and mRNA of osteogenically differentiated mesenchymal stem cells in ankylosing spondylitis. J Rheumatol 43: 1523-1531, 2016.

9. Li X, Chai W, Zhang G, Ni M, Chen J, Dong J, Zhou Y, Hao L, Bai Y and Wang Y: Down-regulation of lncRNA-AK001085 and its influences on the diagnosis of ankylosing spondylitis. Med Sci Monit 23: 11-16, 2017.

10. Wang H, Zhao J, Zhang H, Huang Y, Wang S, Tu Q and Yang N: CARD11 blockade suppresses murine collagen-induced arthritis via inhibiting CARD11/Bcl10 assembly and T helper type 17 response. Clin Exp Immunol 176: 238-245, 2014.

11. Mahurkar S, Moldovan M, Suppiah V and O'Doherty C: Identification of shared genes and pathways: A comparative study of multiple sclerosis susceptibility, severity and response to interferon beta treatment. PLoS One 8: e57655, 2013.

12. O'Brien SA, Zhu M and Zhang W: The importance of IL-6 in the development of LAT-mediated autoimmunity. J Immunol 195: 695-705, 2015. 
13. Aslani S, Mahmoudi M, Garshasbi M, Jamshidi AR, Karami J and Nicknam MH: Evaluation of DNMT1 gene expression profile and methylation of its promoter region in patients with ankylosing spondylitis. Clin Rheumatol 35: 2723-2731, 2016.

14. Langfelder $P$ and Horvath S: WGCNA: An R package for weighted correlation network analysis. BMC Bioinformatics 9: 559, 2008.

15. Jiang W, Liu Y, Liu R, Zhang $\mathrm{K}$ and Zhang Y: The lncRNA DEANR1 facilitates human endoderm differentiation by activating FOXA2 expression. Cell Rep 11: 137-148, 2015.

16. Wang P, Ning S, Zhang Y, Li R, Ye J, Zhao Z, Zhi H, Wang T, Guo Z and Li X: Identification of lncRNA-associated competing triplets reveals global patterns and prognostic markers for cancer. Nucleic Acids Res 43: 3478-3489, 2015.

17. Fei Q, Bai X, Lin J, Meng H, Yang Y and Guo A: Identification of aberrantly expressed long non-coding RNAs in postmenopausal osteoporosis. Int J Mol Med 41: 3537-3550, 2018.

18. Chen WC, Wei CC, Lu HF, Wong HS, Woon PY, Hsu YW, Huang JD and Chang WC: rs657075 (CSF2) is associated with the disease phenotype (BAS-G) of ankylosing spondylitis. Int J Mol Sci 18: E83, 2017.

19. Wang L, Chen Z, An L, Wang Y, Zhang Z, Guo Y and Liu C: Analysis of long non-coding RNA expression profiles in non-small cell lung cancer. Cell Physiol Biochem 38: 2389-2400, 2016.

20. Peng W and Jiang A: Long noncoding RNA CCDC26 as a potential predictor biomarker contributes to tumorigenesis in pancreatic cancer. Biomed Pharmacother 83: 712-717, 2016.

21. Hirano T, Yoshikawa R, Harada H, Harada Y, Ishida A and Yamazaki T: Long noncoding RNA, CCDC26, controls myeloid leukemia cell growth through regulation of KIT expression. Mol Cancer 14: 90, 2015.

22. Wang S, Hui Y, Li X and Jia Q: Silencing of lncRNA-CCDC26 restrains the growth and migration of glioma cells in vitro and in vivo via targeting miR-203. Oncol Res 26: 1143-1154, 2018.

23. Xu H, Liew LN, Kuo IC, Huang CH, Goh DL and Chua KY: The modulatory effects of lipopolysaccharide-stimulated B cells on differential T-cell polarization. Immunology 125: 218-228, 2010.

24. Huan J, Kaler LJ, Mooney JL, Subramanian S, Hopke C, Vandenbark AA, Rosloniec EF, Burrows GG and Offner H: MHC class II derived recombinant $\mathrm{T}$ Cell receptor ligands protect DBA/1LacJ mice from collagen-induced arthritis. J Immunol 180: 1249-1257, 2008.

25. Landers-Ramos RQ, Sapp RM, Jenkins NT, Murphy AE, Cancre L, Chin ER, Spangenburg EE and Hagberg JM: Chronic endurance exercise affects paracrine action of CD31 ${ }^{+}$and $\mathrm{CD} 34+$ cells on endothelial tube formation. Am J Physiol Heart Circ Physiol 309: H407-H420, 2015.

26. Azizi E, Massoud A, Amirzargar AA, Mahmoudi M, Soleimanifar N, Rezaei N, Jamshidi AR, Nikbin B and Nicknam MH: Association of CTLA4 gene polymorphism in Iranian patients with ankylosing spondylitis. J Clin Immunol 30 268-271, 2010.
27. Yang PT, Kasai H, Zhao LJ, Xiao WG, Tanabe F and Ito M: Increased CCR4 expression on circulating CD4(+) T cells in ankylosing spondylitis, rheumatoid arthritis and systemic lupus ery thematosus. Clin Exp Immunol 138: 342-347, 2004.

28. Jandus C, Bioley G, Rivals JP, Dudler J, Speiser D and Romero P: Increased numbers of circulating polyfunctional Th17 memory cells in patients with seronegative spondylarthritides. Arthritis Rheum 58: 2307-2317, 2008.

29. Keir ME, Butte MJ, Freeman GJ and Sharpe AH: PD-1 and its ligands in tolerance and immunity. Annu Rev Immunol 26: 677-704, 2008.

30. Shinohara T, Taniwaki M, Ishida Y, Kawaichi M and Honjo T: Structure and chromosomal localization of the human PD-1 gene (PDCD1). Genomics 23: 704-706, 1994.

31. Bertin J, Wang L, Guo Y, Jacobson MD, Poyet JL, Srinivasula SM, Merriam S, DiStefano PS and Alnemri ES: CARD11 and CARD14 are novel caspase recruitment domain (CARD)/membrane-associated guanylate kinase (MAGUK) family members that interact with BCL10 and activate NF-kappa B. J Biol Chem 276: 11877-11882, 2001.

32. Chabaud M, Fossiez F, Taupin JL and Miossec P: Enhancing effect of IL-17 on IL-1-induced IL-6 and leukemia inhibitory factor production by rheumatoid arthritis synoviocytes and its regulation by Th2 cytokines. J Immunol 161: 409-414, 1998.

33. Maddur MS, Miossec P, Kaveri SV and Bayry J: Th17 cells: Biology, pathogenesis of autoimmune and inflammatory diseases, and therapeutic strategies. Am J Pathol 181: 8-18, 2012.

34. Yan P, Luo S, Lu JY and Shen X: Cis- and trans-acting lncRNAs in pluripotency and reprogramming. Curr Opin Genet Dev 46: 170-178, 2017.

35. Pian L, Wen X, Kang L, Li Z, Nie Y, Du Z, Yu D, Zhou L, Jia L, Chen N, et al: Targeting the IGF1R pathway in breast cancer using antisense lncRNA-mediated promoter cis competition. Mol Ther Nucleic Acids 12: 105-117, 2018.

36. Lipovich L: Abstract IA3: Regulatory networks in onco-IncRNAomics: Cis-regulation and non-conservation. Indian J Microbiol 52: 400-405, 2012.

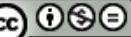

This work is licensed under a Creative Commons Attribution-NonCommercial-NoDerivatives 4.0 International (CC BY-NC-ND 4.0) License. 